\title{
Photo-stimulation effect on the human EEG alpha-wave
} spectrum

\author{
Ryozo Aoki $^{1}$, Yasuyuki Sonezaki ${ }^{2}$, Tahei Kitamura ${ }^{2}$, Kazuhiro Satoh ${ }^{3}$ \\ ${ }^{1}$ Department of Physiology, Osaka University Graduate School of Medicine, Yamadaoka, Japan \\ ${ }^{2}$ Department of Electrical \& Electronics Engineering, College of Industrial Technology, Amagasaki, Japan \\ ${ }^{3}$ College of Business Administration and Economics, Aomori Public College, Aomori City, Japan \\ Email: ryo-aoki@kcc.zaq.ne.jp
}

Received 6 February 2013; revised 26 March 2013; accepted 30 April 2013

Copyright (C) 2013 Ryozo Aoki et al. This is an open access article distributed under the Creative Commons Attribution License, which permits unrestricted use, distribution, and reproduction in any medium, provided the original work is properly cited.

\begin{abstract}
The EEG $\alpha$ wave mode shows chaotic characters and the frequency spectrum is entrained to the external photo-stimulation peak. This effect was observed exceedingly in the photo-sensitive children as compared with the normal adults. The $\alpha$ spectrum shows asymmetric components with lower frequency-side tail. This spectrum shape could be realized from the computation in terms of the McCulloch-Pitts model and presented in comparison with the observed result. From this analysis, it turns out that the frequency spectrum analysis is most essential for the investigation of the EEG characteristics in comparison with simple waveform inspections in the time-passage. When light flashing frequencies come close to the alpha peak, the both peaks are fused in a giant single peak. These phenomena cannot be understood by the simple mechanical resonant theory but as discussed from the viewpoint of the soft chaotic dynamics of the neural network. Here the both peak intensities $I_{a}$ and $I_{\mathrm{ex}}$ are investigated under different conditions of $\omega_{\mathrm{ex}}<\omega_{a}$, and $\omega_{a}<\omega_{\mathrm{ex}}$, and it is shown that the entrainment effect is remarkably different in both cases. This result can be understood from the relating neuronal numbers and discussed.
\end{abstract}

Keywords: EEG; Alpha-Wave; Frequency Spectrum; Light Flashing Stimulation; Chaotic Dynamics;

Spectrum Entrainment

\section{INTRODUCTION}

Recently human brain neural networks have been paid much attention from the view point of the information treatment process $[1,2]$.

In the course of biological evolution well developed animals have neural networks of brain and they show a self-sustained electromagnetic oscillations of the EEG, and one of the authors Aoki previously reported an observation of the EEG at the most primitive invertebrate planarian [3] where its frequency spectrum was found in a broad continuum differently from specific peak character of the human being, probably as due to the substantial feed-back effect of the neural net-work [3].

Human EEG presents several patterns e.g. alpha- betaand delta-waves, following to the mental situations and alpha wave corresponds to the rest state in comfort, and shows frequency spectrum curve of one or two sharp peaks at $8-13 \mathrm{~Hz}$ accompanied with some tail in the lower frequency side.

Regarding the generating mechanism of this EEG, successive firing of synaptic neuronal network has been discussed in terms of the coupling synapse-number, and strength, and the firing threshold level [3]. Accordingly, another author Satoh set up a scheme based on the McCulloch-Pitts model and succeeded in calculation of the alpha spectrum feature [4].

For participants under EEG observations, photo stimulation of repeating flash-light is applied and brings a response to the EEG spectrum through the retina and optic nerve.

In case of the mechanical circuits, superposing of both oscillations takes place and resonates only just at the same frequency, however the neuronal system shows more soft characteristics as expressed by the chaotic dynamics [5]. That is when the light flashing frequency; $\omega_{\mathrm{ex}}$ approaches near to the intrinsic alpha peak frequency; $\omega_{\alpha}$, the alpha peak component is entrained to the light flashing spectrum peak. As the result, alpha peak intensity $\mathrm{I}_{\alpha}$ is weakened and the stimulation peak intensity $\mathrm{I}_{\mathrm{ex}}$ is enhanced. Those characteristics are here reported with a remarkable spectrum change. 


\section{EXPERIMENTS}

For the participants, 5 numbers of normal sensitive adults (male students; 19 - 32 years), and 10 photo-sensitive children ( 7 - 15 years; previously experienced discomfort by observing flashing TV display and inquiring to the hospital) were adopted, and the EEG observations were undertaken at the inspection laboratory of the hospital (the Seikei-kai H., the Osaka Prefectural H. and the Sakai Municipal H.).

Before this experiment with light flashing, it was informed that if the participant feels any occurrence of discomfort soon it should be reported to the operator, then the observation would be stopped at once and appropriate care would be applied. This was consented by all the participants.

The equipment system of this EEG observation is presented schematically in Figure 1.

$\mathrm{The} \mathrm{Ag} / \mathrm{AgCl}$ electric terminals were attached onto the head of the participants, at the $\mathrm{O}_{2}$ site and the right ear lobe for the monopole-induction $(<500 \mathrm{~K} \cdot \mathrm{Ohm})$. After processing with usual EEG waveform analyzer with a band-pass filter of $1 \mathrm{~Hz} \sim 40 \mathrm{~Hz}$, the signal was stored in a digital recorder (Sony Precision Technology Inc. \#PC204Ax; $0-20 \mathrm{kHz}$ ), and then introduced to a digital FFT analyzer (Advantest Co. Japan digital spectrum analyzer \#TR9407) for frequency spectrum analysis.

For observation of the photo-stimulation effect, flashing light (white) was illuminated from $30 \mathrm{~cm}$ above the closed eyelid. The stimulation was continued 1 minute with 1 minute interval for each $1 \mathrm{~Hz}$ step of the $1 \sim 20 \mathrm{~Hz}$ frequency range.

\section{RESULTS}

\subsection{Intrinsic EEG Spectrum without Photo Irradiation}

The every five normal participants showed a similar frequency spectrum as shown in Figure 2 (upper), which presented a sharp single peak $\omega_{\alpha}$, and the photo-sensitive participants showed rather broad peak with some plateau tail at lower frequency site of $7-10 \mathrm{~Hz}$ as shown in Figure 2 (lower).

\subsection{EEG Spectrum Observed under Flashing Illuminations}

With use of the flashing light apparatus in Figure 1, photo stimulation was applied and the repetition frequency $\omega_{\mathrm{ex}}$ was changed step-likely in the range of $1-19 \mathrm{~Hz}$, and the relevant EEG spectra are observed as shown in Figure 3. Here to be noted is that at the corresponding frequency $\omega_{\mathrm{ex}}$, a sharp peak appears independently from the intrinsic $\omega_{\alpha}$ peaks when they are well apart from each other. Now the $\omega_{\mathrm{ex}}$ is increased from the lower site and near to the $\omega_{\alpha}$ an interaction takes place between the both components.

Different from the human being, we have also observed similar kind of effect in planaria, which has sensitivity not to light but skin vibrations due to water waves, and the EEG has been observed under skin vibration stimulation, and similarly accompanied with sharp line spectrum of the vibrations [3].

As is shown in Figure 3 (left, and right) the $\omega_{\alpha}$ components are entrained and its peak height $\mathrm{I}_{\alpha}$ is depressed,

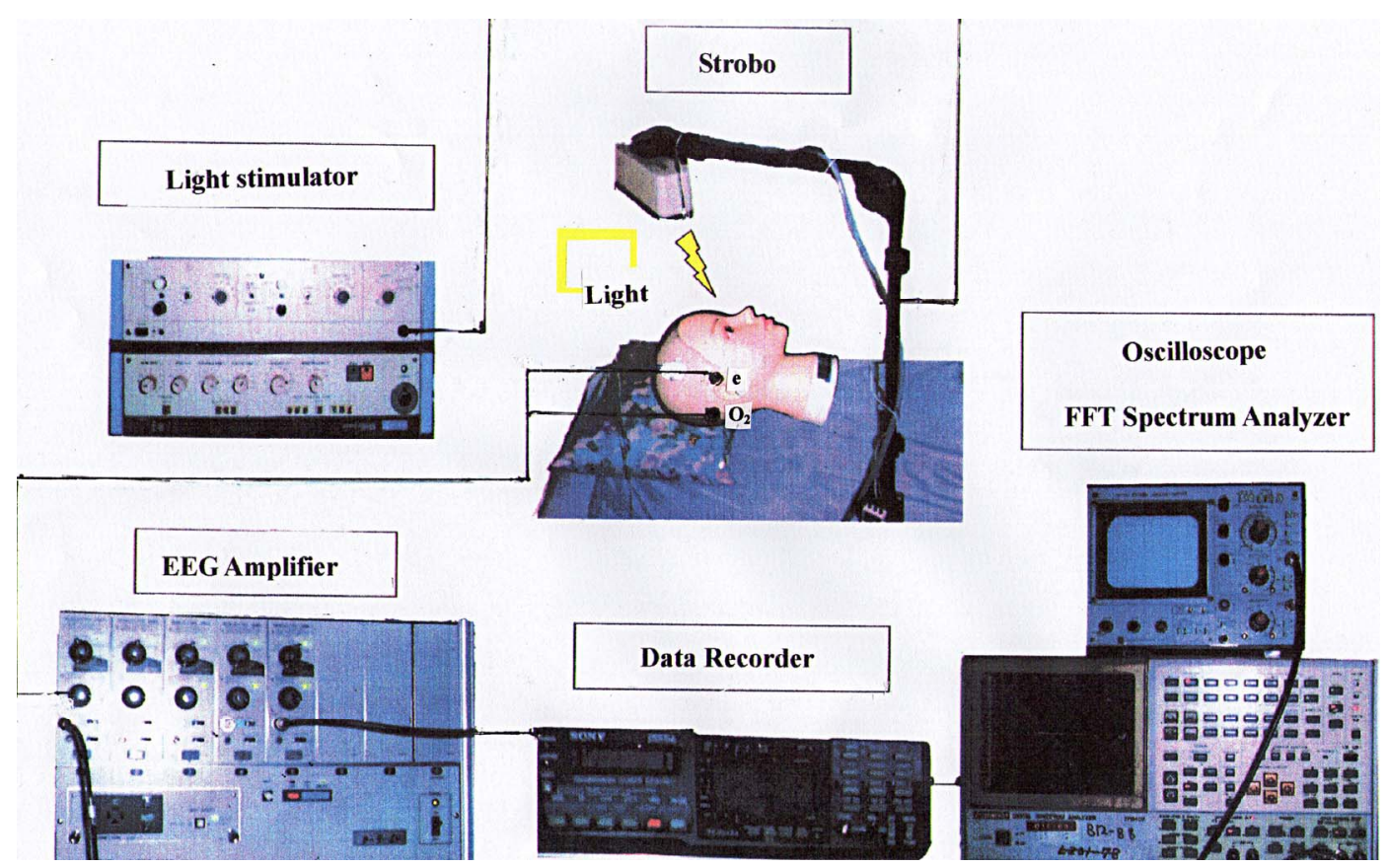

Figure 1. Diagram of the EEG observation equipments under photo-stimulation. 

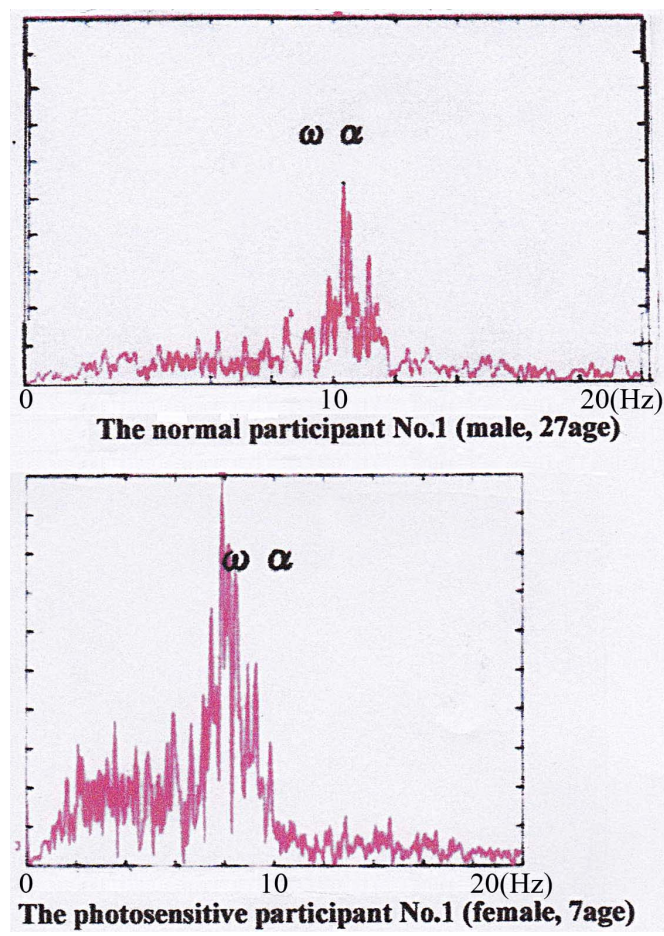

Figure 2. Intrinsic EEG frequency spectrum.

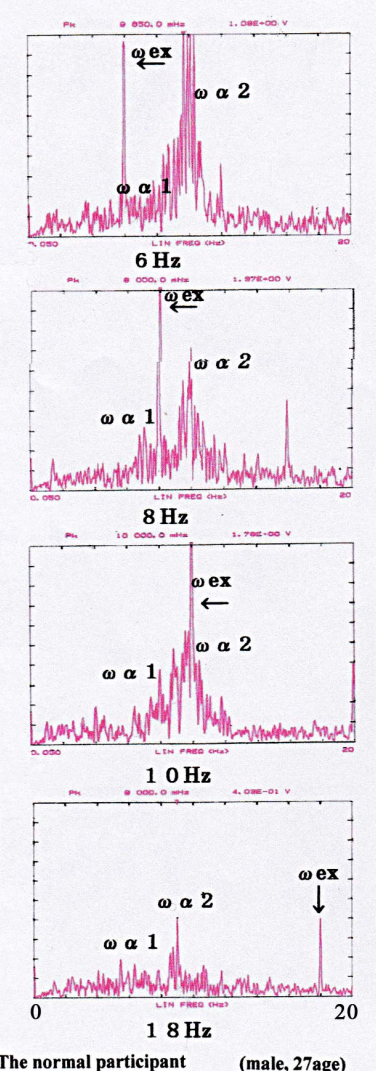

(a)

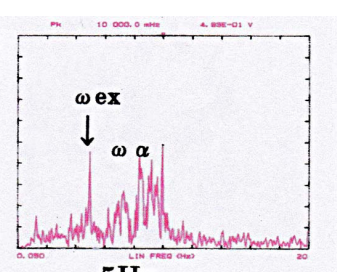

$5 \mathrm{~Hz}$
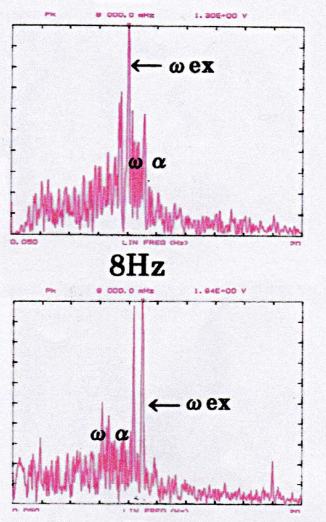

$9 \mathrm{~Hz}$

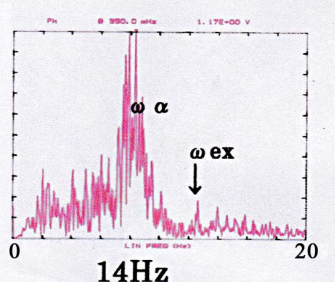

$14 \mathrm{~Hz}$

(b)
Figure 3. EEG frequency spectrum under flashing stimulation. and in exchange, the corresponding height $\mathrm{I}_{\mathrm{ex}}$ is increased. When they come near closer, it goes into a giant peak, and the peak-frequency is not $\omega_{\alpha}$ but the $\omega_{\mathrm{ex}}$.

Moreover, when $\omega_{\text {ex }}$ exceeds beyond the $\omega_{\alpha}$, the entrainment effect decreases rapidly, and the peak height $\mathrm{I}_{\alpha}$ recovers as is shown in Figures 3(a) and (b). This asymmetric character between the range of $\omega_{\mathrm{ex}}<\omega_{\alpha}$ and $\omega_{\alpha}<$ $\omega_{\mathrm{ex}}$ is one of the most remarkable point of this report.

In order to express this result clearly, the peak heights $\mathrm{I}_{\mathrm{ex}}$ and $\mathrm{I}_{\alpha}$ are plotted against $\omega_{\mathrm{ex}}$ in Figure 4. Here we recognize that as goes the entraining, $\mathrm{I}_{\alpha}$ decreases and $\mathrm{I}_{\mathrm{ex}}$ increases alternatively, and this effect is more significant at $\omega_{\text {ex }}<\omega_{\alpha}$.

In comparison among the photo-sensitive's in Figures $\mathbf{2}$ and $\mathbf{3}$ and the normal's in Figures $\mathbf{4}$ and 5, it turns out that the effect is more significant in the photo-sensitive children, and the normal adults show rather slightly and limited only when $\omega_{\mathrm{ex}}$ comes from the lower side and well close to $\omega_{\alpha}$.

\section{DISCUSSION}

As for the EEG spectrum, one of the authors, K. Satoh has investigated the characteristics from the view point of a brain neural net-work model [6], and attempted to compute an intrinsic pulse propagation along the neurons through the synaptic junctions having various connection parameters, and succeeded in realizing a self excited oscillation mode having a similar frequency spectrum pattern with the $\alpha$-wave [4], by setting a couple of many parameters. e.g., total number of the neurons $\mathrm{N}=10^{3}$ in the oscillating zone, average number of the synaptic junctions $n=10$ of each neuronal cell, the action potential $\mathrm{W}=1.67$, and the refractory period $\mathrm{q}=3$.
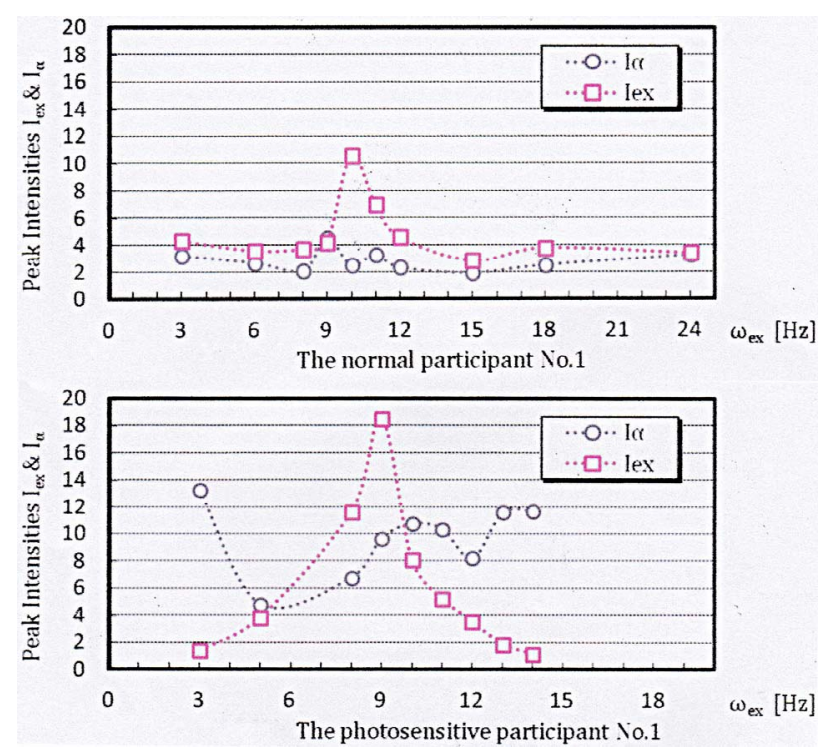

Figure 4. The EEG spectrum peak heights $\mathrm{I}_{\mathrm{ex}} \& \mathrm{I}_{\alpha}$ with flashing frequency $\omega_{\mathrm{ex}}$. 


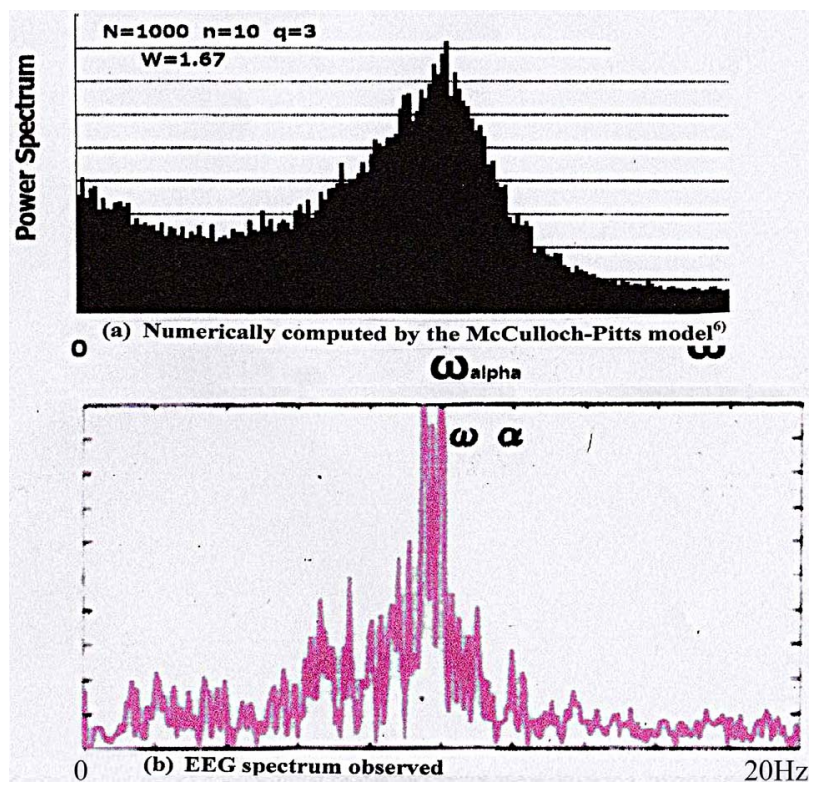

Figure 5. Comparison of the EEG alpha spectrum between the calculated (a) and the observed (b).

As the parameter $\mathrm{N}, \mathrm{W}$ and the $\mathrm{q}$ become large, the firing continues go and back, and then he succeeded to obtain a self sustaining stationary oscillation mode [6]. Moreover a similar pattern of the alpha spectrum was realized [4] as shown in Figure 5, which is characterized by an asymmetric component in the peak and also by the low frequency side continuum spectrum.

In Figure 3, we can observe that the spectrum component is enhanced in the lower frequency side. This character is also seen in the intrinsic $\alpha$ spectrum in Figure 2, which shows also the low frequency side tail and the asymmetric shape of the peak.

As for the reason of this character, it is considered that in the low frequency $\omega$ range, the firing period $\tau=1 / \omega=$ $\mathrm{N} \delta \mathrm{t}$ (where intrinsic synapse transit time $\delta \mathrm{t}$ is as small as $1 \sim 2 \mathrm{msec}$ ) is long, and then the oscillating frequency $\omega$ range is lowered by the large $\mathrm{N}$ of the regarding neuronal region in the brain [3].

The cause of this low-frequency side advantage in the entrainment is understood as follows. For the lower frequency range, the synapse numbers regarding the self oscillating mode is enlarged and the relevant firing region contains large number of the synapse network.

On the other hand, in the higher frequency range the containing synapse number is smaller and the sustaining oscillation is some limited in a local region with smaller synaptic numbers, and then less stable by the fluctuations.

From this view point, the low frequency side advantage in the entrainment effect, and the enhancement of the lower-side spectrum $\left(\omega_{\mathrm{ex}}<\omega_{\alpha}\right)$ as seen in Figures 2-4 can be understood.
In comparison, the higher frequency of $\left(\omega_{\alpha}<\omega_{\mathrm{ex}}\right)$ range, the number $\mathrm{N}$ is rather small, and any stable mode relating to the small loop or limit cycle in the phase space will be difficult to be well maintained.

When the synaptic junction number $\mathrm{n}$ goes to large, the synaptic connections become multi-dimensionally entangled and the oscillating loop [7] approaches shorter due to e.g. surface mode of the volumetric region, which brings effective number as small as $\mathrm{N}^{2 / 3} \sim 10^{2}$. In that case, the frequency $\omega=(\mathrm{N} \delta \mathrm{t})^{-1}=10 \mathrm{~Hz}$ is obtained and it agrees well with the observed $\alpha$-wave frequency of $8-13 \mathrm{~Hz}$.

In case of the alpha wave spectrum having two peaks as shown in Figure 3, it may be considered as due to the sub-domain structure in the real synapse region, or double strange attractors in the chaotic phase-space [5], and then the corresponding point is jumping go and back among them. In case of these meta-stable state, the $\omega_{\alpha}$ can be easily entrained by any stimulation of $\omega_{\text {ex }}$. Really these two-peaks $\alpha$ spectrum can be sometimes observed in the photo-sensitive patients.

The $\alpha$-waves in EEG can be observed usually in the rest state of comfort, and has been paid attention from the view point of mental health [8]. Usually the EEG is surveyed by the time sweep waveforms as shown in Figure 6, where we can observe almost regular 10 waves in the one second sweeping range. Really in this case, the object is comfortable by hearing natural rhythmical sound in the rest state. However, the EEG frequency spectrum analysis reveals that an entrainment of the $\alpha$ component $\omega_{\alpha}$ takes place to the extrinsic stimulation peak $\omega_{\text {ex }}$ as seen in Figure 6(b). it is worthy to note that the prominent regular waves in Figure 6(a) of time sweep scale dominantly comes from the stimulation $\omega_{\mathrm{ex}}$ but not by the intrinsic $\alpha$-component which may supposed from the waveform of Figure 6(a). From this result, it should be commented that for the EEG investigations, the frequency spectrum analysis must be considered as essentially important $[2,8]$.

\section{CONCLUSIONS}

1) In comparison of a computational result with the observed EEG alpha-spectrum, we could get a reasonable agreement. Accordingly the EEG wave generating mechanism in the brain neural network was revealed out on the basis of the McCulloch-Pitts model.

2) 10 photo-sensitive children who have shown discomfort in observation of the flashing TV display were inspected together with 6 normal adult participants, and it turns out that the photosensitive participants showed remarkable entrainment effect of the $\omega_{\alpha}$ peak to the photo irradiation peak $\omega_{\mathrm{ex}}$ even well after the discomfort shock, in the EEG frequency spectrum analysis.

3) The spectrum peak entrainment effect was observed 


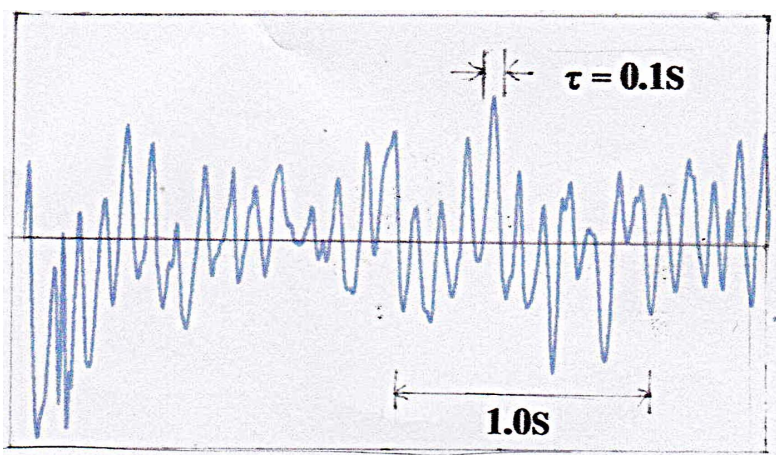

(a) Usual EEG time-scale flowchart.

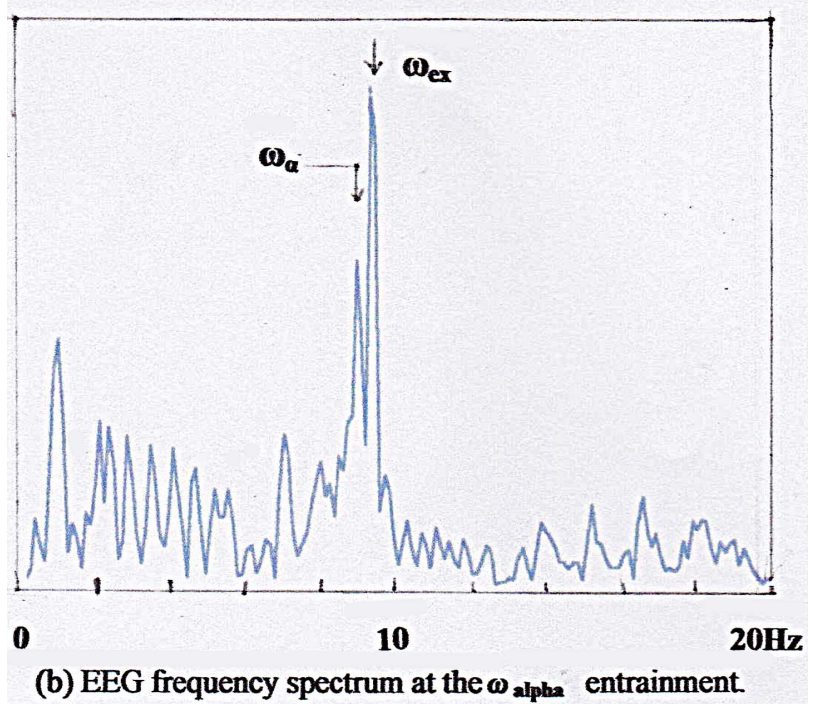

Figure 6. Comparison of EEG timescale flowchart (a) and the frequency spectrum (b) under flashing stimulation of $\omega=9.8$ $\mathrm{Hz}$.

significantly in the lower frequency side of the $\alpha$-peak. This asymmetric character could be understood from the chaotic view point of the self-sustaining neuronal oscillation mode in the entangled synaptic network.

\section{ACKNOWLEDGEMENTS}

In this investigation, the EEG observations were carried out in the inspection laboratories of the following hospitals; the Osaka Prefectural H. the Sakai Municipal H., and the Osaka Seikei-kai H. and the data recording were kindly undertaken by Dr. H. Maekawa (Osaka Prefect. H), Dr. Yosiki Morita (Sakai Munis, H.), and also Dr. Takesi Tizaki (Osaka Seikei, H.). The authors are gratefully thanks for their kind efforts and hospitality.

\section{REFERENCES}

[1] Grill-Spector, K., Henson, R. and Hartin, A. (2006) Repetition and the brain neural models of stimulus-specific effects. Trends in Cognitive Sciences, 10, 14-23. doi:10.1016/j.tics.2005.11.006

[2] Moruzzi, G. and Magueen, H.W. (1999) Brain stem reticular formation and activation of the EEG. Electroencephalography and Clinical Neuro Physiology, 1, 455473.

[3] Aoki, R., Wake, H., Sasaki, H. and Agata, K. (2009) Recording and spectrum analysis of the planarian electroencephalogram. Neuro Science, 159, 908-914. doi:10.1016/i.neuroscience.2008.11.011

[4] Satoh, K. (1989) Computor experiment on characteristic modes of excitation in a random neural network on the mcculloch-pitts model. Journal of the Physical Society of Japan, 58, 2741-2757. doi:10.1143/JPSJ.58.2741

[5] Babloyantz, A., Salazar, J.M. and Nicolis, C. (1985) Evidence of chaotic dynamics of brain activity during the sleep cycle. Physics Letters, 111A, 152-156.

[6] Satoh, K. (1990) Rhythmic activity in a random neural neural network model. Journal of the Physical Society of Japan, 59, 4297-4301. doi:10.1143/JPSJ.59.4297

[7] Aoki, R., Miyashita, T., Katoh, H. and Ueno, S. (1987) Chaotic features in EEG of human brain under photic flicker stimulations. Proceedings of the 6th International Conference on Biomagnetism, Tokyo, 1987, 226-229.

[8] Holczberger, M., et al. (2012) Electroencephalographic coherence during emotional identification task. World Journal of Neuroscience, 2, 24037. 